\title{
Development of Cytogenetic Abnormalities in Myelodysplastic Syndromes
}

\author{
Bandara WMMS $^{1,2}$, Rathnayake AJIS ${ }^{1,2}$, Goonasekera HWW ${ }^{1}$ and Dissanayake VHW ${ }^{{ }^{*}}$
}

${ }^{1}$ Human Genetics Unit, Faculty of Medicine, University of Colombo, Sri Lanka

${ }^{2}$ Department of Pre-clinical Sciences, Faculty of Medicine, General Sir John Kotelawala Defence University, Rathmalana, Sri Lanka

"Corresponding author: Vajira H.W. Dissanayake, The Human Genetics Unit, Faculty of Medicine, University of Colombo, 25, Kynsey Road, Colombo 8, Sri Lanka, Tel: 0777351835; Fax: 0112691581; E-mail: vajirahwd@hotmail.com

Received date: March 08, 2016; Accepted date: May 20, 2016; Published date: May 25, 2016

Copyright: (c) 2016 Bandara WMMS, et al. This is an open-access article distributed under the terms of the Creative Commons Attribution License, which permits unrestricted use, distribution, and reproduction in any medium, provided the original author and source are credited.

\begin{abstract}
Myelodysplastic syndromes (MDS) are a heterogeneous group of clonal hematopoietic stem cell disorders characterized by ineffective hematopoiesis resulting in cellular dysplasia and peripheral cytopenias. Research into MDS have found that both hematopoietic stem cells (HSCs) and mesenchymal stem cells (MSCs) in the nurturing niche of HSCs are genetically altered in MDS. In this review we present the existing views on the origin of cytogenetic abnormalities in HSC and MSC compartments in MDS. Based on the available data, we speculate that the origin of the chromosomal aberrations of the hematopoietic compartment occurs at the hematopoietic stem/ progenitor level. The genetic aberrations in MSC compartment appears to initiate independently from their hematopoietic counterparts. Whether the genetic events in both HSC and MSC compartments which lead to MDS occur simultaneously or at different time points in the disease development need to be determined in future.
\end{abstract}

Keywords: Myelodysplastic syndromes; Hematopoietic stem cells; Mesenchymal stem cells; Cytogenetics

\section{Introduction}

Myelodysplastic Syndromes (MDS) result from malignant transformation of a HSC, which has growth advantage over normal HSCs resulting in an abnormal clone of cells [1,2]. Chromosomal abnormalities are detected in approximately $30 \%$ to $70 \%$ of MDS patients and believed to have a strong correlation with the disease origin and progression [2]. Bone marrow (BM) microenvironment which is the nurturing niche of HSCs is also known to be altered in MDS [3,4]. Research has found that mesenchymal stem cells (MSCs), which give rise to most of the stromal components of the marrow is altered in MDS $[4,5]$.

The genetic abnormalities present in MDS are thought to initiate at the level of stem cells in the hematopoietic and mesenchymal compartments $[5,6]$. These genetic abnormalities occur due to previous exposure to mutagens like benzene, alkylating agents, gasoline, topoisomerase II inhibitors, radiation etc. [7-13]. Due to exposure to insecticides, pesticides and herbicides agricultural workers are reported to have higher incidence of developing MDS [7]. Hazardous heterocyclic aromatic and aliphatic organic chemicals, benzene derivatives and complex compounds with nitrogen, sulphur and oxygen present in these insecticides and pesticides are identified as disease initiating agents $[7,8]$. Several studies describe alcohol as a potential risk factor for development of MDS. Although the exact link between alcohol and MDS is not yet clear, it is reported that alcoholics induce chromosomal abnormalities in hematopoietic cells with altered gene expression patterns and adverse effects on the immune system $[9,10,11]$. Tobacco products like cigarettes which contain benzene and other carcinogens also induce chromosomal aberrations in primitive bone marrow cells leading to development of MDS $[9,12,13]$.

Genetically abnormal HSCs later differentiate into morphologically and functionally abnormal myeloid lineages [6] while genetically abnormal MSCs may contribute to propagating the abnormal clones of HSCs leading to disease progression $[6,14]$.

\section{The role of bone marrow stroma and mesenchymal stem cells in MDS}

Hematopoietic cell-centered approach of explaining MDS describes the origin of disease as a neoplastic transformation of hematopoietic stem/progenitor cells [6]. However difficulties in duplicating the pathological features of the disease by simple transplantation of hematopoietic stem cells to immunodefficient mice models have highlighted the involvement of the abnormal marrow microenvironment in the disease origin and progression with pivotal yet unexplained role for marrow stroma $[14,15]$. Research data also supports the view that abnormal microenvironment selectively supports the expansion of the malignant clone through altered signaling between diseased HSCs and malfunctioning MSCs [16-21]. Impaired capacity of MSCs to support normal hematopoiesis in MDS is attributed to abnormal signaling, altered interactions with the hematopoietic cells, altered proliferative and differentiation potential, changes in epigenetic regulation and abnormalities in cytokine secretion [16-21]. Gene expression analyses of MDS derived MSCs have shown altered RNA levels in both canonical and non-canonical WNT signaling pathways and is thought to be associated with impaired proliferative potential of MDS derived MSCs [20]. Geyh et al. in 2013 showed that MSCs derived from all MDS subtypes exhibit decreased growth and proliferative capacities with premature replicative cellular senescence with altered expression in Osteopontin, Jagged1, Kit-ligand and Angiopoietin which involve in the interaction with hematopoietic lineages [21].

The immune-modulatory properties, cytokine secretion and cytogenetic profiles of MSCs have shown to be altered in MDS [16-23]. The groups which claim the genetic susceptibility of MSCs argue on the potential involvement of these cells in the pathogenesis of the disease [16-22]. In spite of data that show MDS-MSCs are functionally 
normal with respect to the ability of sustaining the growth of $\mathrm{CD} 34^{+}$ cells in co-cultures and secretion of normal levels of cytokines and growth factors (TNF- $\alpha$, IL-6, stem cell factor, GM-CSF, VEGF, collagen and fibronectin) $[24,25]$ some other studies show that MDS-MSCs are unable to support hematopoiesis [16-23]. Further, some studies have shown that the MSCs of MDS secrete increased levels of certain cytokines such as TNFa, IL-6, IFN- $\gamma$ and angiogenic cytokines $[17,18]$.

The recently emerged evidence on "oncogenesis in hematopoietic system" reveals that unfavorable alterations in the BM microenvironment can initiate malignant transformation of other cells [26]. With the disease progression, MDS blasts become less apoptotic with increased proliferative capacity, producing phenotypically immature clone of cells, whereas the T-cell compartment shows decreased proliferation and increased apoptosis [27-32]. Kondo et al. in 2010 showed that the increased production of IFN- $\gamma$ and TNFa enhances the proliferation of blasts through the expression of $\mathrm{B} 7-\mathrm{H} 1$ on MDS blasts and could be an early event in the disease progression [33]. Additionally, $\mathrm{B} 7-\mathrm{H}^{+}$blasts suppress proliferation of $\mathrm{T}$ cells and induce their apoptosis in in vitro co-cultures [33]. Moreover, studies on regulatory T cells (Tregs) have shown an altered Treg compartment in lower risk MDS patients [34]. An increase in the number of $\mathrm{CD} 4{ }^{+} \mathrm{FoxP} 3^{+} \mathrm{CD} 25^{+} \mathrm{CD} 127^{\text {low }} \mathrm{CD} 45 \mathrm{RA}^{-} \mathrm{CD} 27^{-}$Tregs is significantly associated with anemia, reduced hemoglobin and increased blast counts [34]. Hence, complex interactions between the abnormal clones of HSCs and the neighboring cells of the marrow microenvironment may modulate the disease development.

\section{The occurrence of cytogenetic abnormalities in MDS- HSCs}

MDS derivation may be a multi step process, but believed to be originated at the hematopoietic stem/progenitor level [6, 19-25]. The cell type which should be considered as anMDS "stem" cell is yet to be disclosed. Whether it is a multipotential stem cell or progenitor cell remains controversial [6,35-39]. The 'mutator stem cell' theory describes that acquired mutations at a critical stage in hematopoiesis act as the basis for additional genetic events during the onset of MDS [36]. Some studies have shown that the disease originates due to an abnormality within the hematopoietic progenitor cell [39]. Hence hematopoietic stem/ progenitor compartment has drawn the central attention of MDS research. MDS is still considered as a true stem cell disorder despite both supportive and oppose evidence for the idea that it may more commonly occur in multipotent myeloid lineages [6,35-39].

Cytogenetic analyses have identified various clonal chromosomal abnormalities in the cells of hematopoietic hierarchy including hematopoietic stem/progenitors as well as in the myeloid and lymphoid lineages of blood cells [35-41]. The common recurring cytogenetic abnormalities in MDS include $\operatorname{del}(5 q),-7 / \operatorname{del}(7 q)$, $-8,-18 /$ del (18q), del (20q),-5,-Y,-17/del (17p) including isochromosome (17q), -21, inv/t(3q), -13/del (13q), -1/del (1q), -21, -11 , del $(12 p), t(5 q)$, del $(11 q)$, and $t(7 q)[1,2,35-43]$. The initiator hematopoietic cell with cytogenetic abnormalities in the hierarchy of hematopoiesis is yet to be identified. Recent studies have shown that $\operatorname{del}(5 \mathrm{q})$ is detected in CD34+CD38-CD90+ (Thy1) HSCs demonstrating that the initiation of deletions in the $5 \mathrm{q}$ occurs at early stage of the hematopoiesis, at a cell with both myeloid and lymphoid potential $[40,31]$. Involvement of non-myeloid lineages is controversial as some research report MDS associated chromosome abnormalities in T-cell, B-cell, or natural killer-cell populations while some other reports claim for the absence of such chromosome aberrations in lymphoid lineages [40,41]. While MDS has very rare chance of evolving into lymphoid acute leukemia, it is noteworthy to indicate that as most of the research have proven; the dysplasia and aberrations are more prominent in cells committed to myeloid lineages [42]. However, some reports argue that only del $5 \mathrm{q}$ could be seen at more primitive HSCs and other aberrations like trisomy 8 might occur at later stages in the hematopoietic hierarchy $[40,43]$. While the controversies still remain the widely accepted hypothesis is that the disease origin occurs at the early stem cell populations [6].

\section{The origin of cytogenetic abnormalities in MDS-MSCs}

The existence of cytogenetic abnormalities in MSCs from patients with MDS is still in debate. Some studies indicate that MSCs derived from MDS are cytogenetically normal [25] while others in their studies have shown that MDS-MSCs show chromosomal abnormalities [24,44-46]. The percentage of presence of structural chromosomal aberrations in MDS derived MSCs is found to be low in some studies $[44,45]$ while there are other reports that claim for higher rates of having abnormal MSCs in MDS [22,46].

Co-existence of genetic aberrations in both hematopoietic and mesenchymal stem cell compartments has been reported by many groups [22, 44-47]. Blau et al. reported that abnormal karyotypes in MSCs can be frequently observed in patients with cytogenetically abnormal HSCs [44]. In such cases with chromosomal abnormalities in MSCs, the reported karyotypes were more complex. However, other groups have observed chromosomal alterations in MSCs of MDS patients with cytogenetically normal HSCs [44-48]. These groups declare that the clonal cytogenetic abnormalities found in MSC populations are mostly numerical and are different from that of their hematopoietic counterparts with no overlap between clonal chromosomal abnormalities of the MSCs from those observed in their HSC counterparts $[22,44,48]$. It is important to highlight that most of the reported cytogenetic abnormalities in MDS-MSCs are not commonly-reported abnormalities of MDS or unique to MDS [49] but may have adverse prognostic impact [23]. Interestingly, no cytogenetic abnormalities have been reported in MSCs from healthy individuals [44-47].

In spite of the existing controversies whether the MDS derived MSCs are cytogenetically abnormal or not, there are many other important questions to be answered. First, it is not yet clear whether the abnormal MSC clones in MDS marrow are derived from the same neoplastic clone. The possible question that needs an answer is whether the same factor (mutagen) that initiate the malignant transformation in HSCs also induce molecular and cytogenetic abnormalities in MSCs. If so, does the mutagen induce same or different genetic alterations in both HSC and MSC compartments? If the origin of genetic alterations in MSCs is independent from HSCs, at what stage of the disease these cells undergo the genetic damage? Answering these questions with existing literature is challenging.

Most of the research up to date support the idea that occurrence of genetic aberrations in MSCs is an independent event, blunting the theory of "neoplastic clonal origin" of genetic aberrations. Many researchers demonstrate that the aberrant MSC clones are present in those with normal HSC karyotypes and when the aberrations co-exist in both HSC and MSC compartments, MDS-MSCs possess distinct chromosomal aberrations than their corresponding hematopoietic counterparts. Yet, the origin or the initial causative of the genetic 
damage may be same but the two cell types develop different chromosomal aberrations after the exposure to the mutagen [22,44]. Two hypothetic models for the origin of cytogenetic aberrations in hematopoietic and mesenchymal stem cell compartments could be put forward as shown in Figure 1.

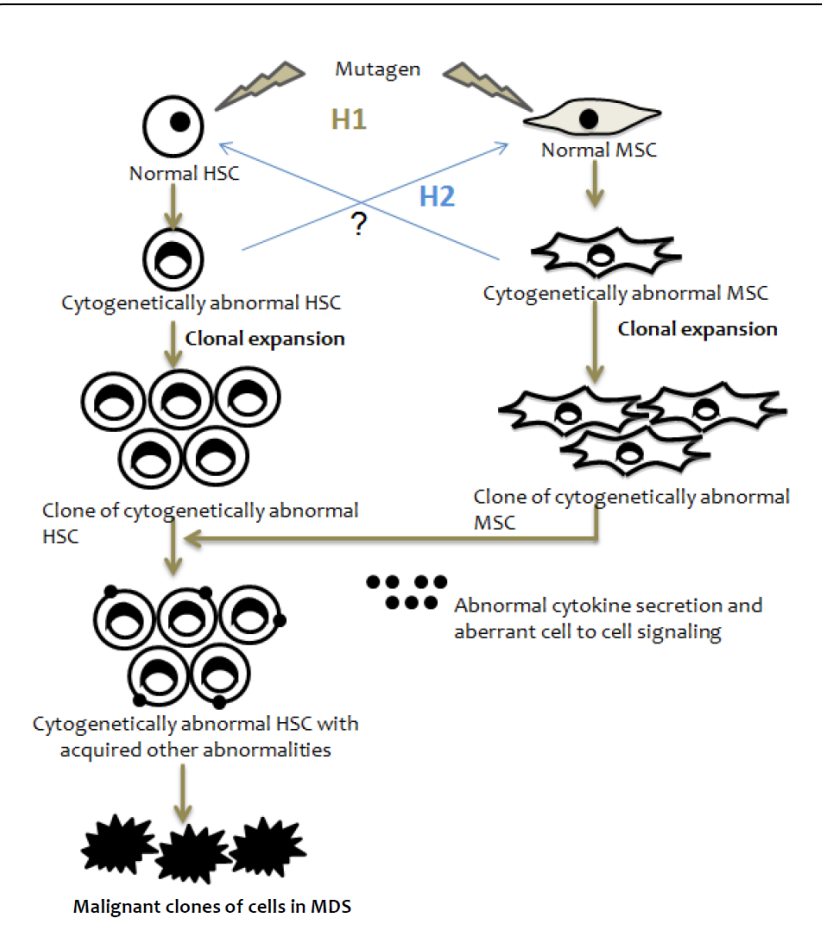

Figure 1: Model for the origin of cytogenetic abnormalities in HSCs and MSCs. Hypothesis 1 (H1): Simultaneous occurrence of genetic abnormalities in both HSC and MSC compartments in MDS. The mutagen (radiation, carcinogenic chemicals, viruses, etc) that initiate the same or different genetic aberrations in both HSC and MSC compartments lead to MDS. Subsequently, the expansion of abnormal HSC clones is supported by the abnormal cytokines and the aberrant cell signaling by abnormal MSC populations. Hypothesis 2 (H2): The aberrations in HSCs or MSCs caused by an initial mutagen may disrupt the integrity of marrow stroma inducing genetic alterations in normal MSCs or normal HSCs respectively leading to development of MDS.

\section{Hypothesis 1}

This hypothesis suggests the origin of genetic aberrations in MSC compartment is initiated independently as an autonomous event (Hypothesis 1 in Figure 1). Whether the genetic events in both HSC and MSC compartments that lead to MDS occur simultaneously or at different time points in the disease development pathway has no clear answer. However, most research leave the possibility of sharing a common genetic modifications by both hematopoietic and mesenchymal compartments which can go undetected by conventional cytogenetic analyses [25,49]. It is also possible that both cell types initiate aberrations independently at the same time period, but the cellular DNA repair systems and other mechanisms of MSCs may prevent occurrence of karyotypic abnormalities in them leading to cytogenetically normal stromal compartment in MDS as shown in some research. It is also possible that both cell types are damaged by the same mutagen but cells can progress chromosomal aberrations independently as it divides leading to additional chromosomal aberrations finally developing a distinct malignant clone with retarded DNA repair systems and genetic instability [50]. Additionally, the higher proliferation rates of HSCs may be a reason for the high incidence of genetic abnormalities in HSC compartment compared to the stromal compartment.

\section{Hypothesis 2}

The cytogenetic aberrations caused by the initial mutagen may occur in one of the compartments (either in HSC or MSC) and the resulting abnormal HSC or MSC clones may induce genetic alterations in the other compartment leading to development of MDS. If MSCs are not altered at the stage of aberrant HSCs development, at what stage of the disease the genetic alterations observed in MSC populations occur, is still inconclusive. Some argue that the genetically altered HSC clones induce the genetic abnormalities in the MSC compartment which develop aberrant MSC clones in marrow stroma (Hypothesis 2 in Figure 1) [14,15]. The defective marrow stroma then secretes the abnormal and altered levels of cytokines leading to the expansion of the abnormal HSC clones resulting progression of MDS $[17-18,33]$. It is also possible that aberrant MSCs that are exposed to a mutagen may induce abnormalities in HSC compartment by aberrant cell to cell signaling and secretion of abnormal factors (Hypothesis 2 in Figure 1).

Therefore, it is worthwhile to consider the evaluation of the genetic integrity of MSCs at different stages of the disease in order to identify the exact point of origin of aberrations in mesenchymal cells. Further, the MDS-derived MSC populations with aberrant clones do not show abnormal phenotypic and growth retardation in in vitro cultures [22,43-46]. Some have reported the functional deficiencies in MDSderived MSCs while others argue that the MDS-MSCs can support normal hematopoiesis [24,25]. It is worth to note that the reported cytogenetic profiles of MSC populations derived from MDS patients consist of normal clones (with normal karyotypes) of MSCs in addition to the genetically aberrant MSC clones. Therefore, one can presume that these normal clones take the growth advantage over the abnormal clones in in vitro cultures or may be in their marrow niche displaying no functional impairment. Hence, analysis of abnormal clones of MDS-MSCs in separation is required to answer the question; whether the abnormal MSCs derived from MDS are functionally normal or not [24]. Further, analyzing genetically aberrant clones in isolation will also allow understanding the contribution and the mechanisms of the genetic abnormalities in relation to the function of MSCs in marrow stroma although the aberrations seem to have no growth advantage over the normal clones of MDS-MSCs [23,24].

\section{Conclusion}

Based on the available data, we believe that the origin of the chromosomal aberrations in MDS occur at the hematopoietic stem/ progenitor level. Further, the origin of genetic aberrations in MSC compartment seems to initiate independently as an autonomous event. Whether the genetic events in both HSC and MSC compartments that lead to MDS occur simultaneously or at different time points in the disease development pathway has no clear answer. Hence, future studies need to be directed towards identifying the stage of origin of genetic events in MSCs and the exact role of genetically aberrant MSC clones in origin/pathogenesis of MDS. Furthermore, whether it is the malignant HSCs reprogram the normal MSCs to an abnormal clone of 
cells which in turn enhances the disease progression or whether the abnormalities in the marrow environment drives the initiating step of MDS is yet to be defined.

\section{References}

1. Kurzrock R (2002) Myelodysplastic syndrome overview. Seminars in Hematology 39: 18-25.

2. Beutler E, Litchman MA, Coller B, Kipps T, Seligsohn U (2001) Willams Hematology. (6thedn), McGrw-Hill medical publishing division, United States.

3. Nombela-Arrieta C, Ritz J, Silberstein LE (2011) The elusive nature and function of mesenchymal stem cells. Nat Rev Mol Cell Biol 12: 126-131.

4. Kastrinaki MC, Pontikoglou C, Klaus M, Stavroulaki E, Pavlaki K, et al. (2011) Biologic characteristics of bone marrow mesenchymal stem cells in myelodysplastic syndromes. Curr Stem Cell Res Ther 6: 122-130.

5. Wang J, Xiao Z (2014) Mesenchymal stem cells in pathogenesis of myelodysplastic syndromes. Stem Cell Investig 1:16.

6. Nimer SD (2008) MDS: a stem cell disorder-but what exactly is wrong with the primitive hematopoietic cells in this disease? Hematology Am Soc Hematol Educ Program 43-51.

7. Nisse C, Haguenoer JM, Grandbastein B, Preudhomme C, Fontaine B, et al. (2001) Occupational and environmental risk factors of the myelodysplastic syndromes in the North of France. Br J Haematol 112: 927-935.

8. West RR, Stafford DA, Farrow A, Jacobs A (1995) Occupational and environmental exposures and myelodysplasia: a case-control study. Leuk Res 19: 127-139.

9. Du Y, Fryzek J, Sekeres MA, Taioli E (2010) Smoking and alcohol intake as risk factors for myelodysplastic syndromes (MDS). Leuk Res 34: 1-5.

10. Obe G, Gobel D, Engeln H, Herha J, Natarajan AT (1980) Chromosomal aberrations in peripheral lymphocytes of alcoholics. Mutat Res 73: 377-386.

11. Datta R, Sherman ML, Kufe DW (1990) Regulation of proto-oncogene and tumor necrosis factor gene expression by ethanol in HL-60 myeloid leukemia cells. Blood 76: 298-301.

12. Bjork J, Albin M, Mauritzson N, Stromberg U, Johansson B, et al. (2000) Smoking and myelodysplastic syndromes. Epidemiology 11: 285-291.

13. Strom SS, Gu Y, Gruschkus SK, Pierce SA, Estey EH (2005) Risk factors of myelodysplastic syndromes: a case-control study. Leukemia 19: 1912-1918.

14. Raaijmakers MH (2014) Disease progression in myelodysplastic syndromes: do mesenchymal cells pave the way? Cell Stem Cell 14 695-697.

15. Medyouf H, Mossner M, Jann JC, Nolte F, Raffel S, et al. (2014) Myelodysplastic cells in patients reprogram mesenchymal stromal cells to establish a transplantable stem cell niche disease unit. Cell Stem Cell 14: 824-837.

16. Aizawa S, Nakano M, Iwase O, Yaguchi M, Hiramoto M, et al. (1999) Bone marrow stroma from refractory anemia of myelodysplastic syndrome is defective in its ability to support normal CD34-positive cell proliferation and differentiation in vitro. Leuk Res 23: 239-246.

17. Kitagawa M, Saito I, Kuwata T, Yoshida S, Yamaguchi S, et al. (1997) Overexpression of tumor necrosis factor (TNF)-alpha and interferon (IFN)-gamma by BM cells from patients with myelodysplastic syndromes. Leukemia 11: 2049-2054.

18. Wetzler M, Kurzrock R, Estrov Z, Estey E, Talpaz M (1995) Cytokine expression in adherent layers from patients with myelodysplastic syndrome and acute myelogenous leukemia. Leuk Res 19: 23-34.

19. Deeg HJ, Beckham C, Loken MR, Bryant E, Lesnikova M, et al. (2000) Negative regulators of hemopoiesis and stroma function in patients with myelodysplastic syndrome. Leuk Lymphoma 37: 405-414.

20. Pavlaki K, Pontikoglou CG, Demetriadou A, Batsali AK, Damianaki A, et al. (2014) Impaired proliferative potential of bone marrow mesenchymal stromal cells in patients with myelodysplastic syndromes is associated with abnormal WNT signaling pathway. Stem Cells Dev 23: 1568-1581.

21. Geyh S, Oz S, Cadeddu RP, Fröbel J, Brückner B, et al. (2013) Insufficient stromal support in MDS results from molecular and functional deficits of mesenchymal stromal cells. Leukemia 27: 1841-1851.

22. Flores-Figueroa E, Arana-Trejo RM, Gutiérrez-Espíndola G, PérezCabrera A, Mayani H (2005) Mesenchymal stem cells in Myelodysplastic Syndromes: phenotypic and cytogenetic characterization. Leuk Res 29: 215-224.

23. Klaus M, Stavroulaki E, Kastrinaki MC, Fragioudaki P, Giannikou K, et al. (2010) Reserves, functional, immunoregulatory and cytogenetic properties of bone marrow mesenchymal stem cells in patients with myelodysplastic syndromes. Stem Cells Dev 19: 1043-1054.

24. Flores-Figueroa E, Montesinosa JJ, Flores-Guzmána P, GutiérrezEspíndolab G, Arana-Trejoc RM, et al. (2008) Functional analysis of myelodysplastic syndromes-derived mesenchymal stem cells. Leuk Res 32: 1407-1416.

25. Soenen V, Kotb R, Bonnet ML, Guillier M, Flamant S, et al. (2001) Mesenchymal cells generated from patients with myelodysplastic syndromes are devoid of chromosomal clonal markers and support shortand long-term hematopoiesis in vitro. Oncogene 24: 2441-2448.

26. Raaijmakers $\mathrm{MH}$ (2011) Niche contributions to oncogenesis: emerging concepts and implications for the hematopoietic system. Haematologica 96: 1041-1048

27. Shioi Y, Tamura H, Yokose N, Satoh C, Dan K, et al. (2007) Increased apoptosis of circulating $\mathrm{T}$ cells in myelodysplastic syndromes. Leuk Res 31: 1641-1648

28. Mufti GJ, Figes A, Hamblin TJ, Oscier DG, Copplestone JA (1992) Immunological abnormalities in myelodysplastic syndromes. I. Serum immunoglobulins and autoantibodies. Br J Haematol 63: 143-147.

29. Parker JE, Mufti GJ, Rasool F, Mijovic A, Devereux S, et al. (2000) The role of apoptosis, proliferation, and the Bcl-2-related proteins in the myelodysplastic syndromes and acute myeloid leukemia secondary to MDS. Blood 96: 3932-3938.

30. Ogata K, Nakamura K, Yokose N, Tamura H, Tachibana M, et al. (2002) Clinical significance of phenotypic features of blasts in patients with myelodysplastic syndrome. Blood 100: 3887-3896.

31. Ogata K, Yokose N, Ito T, An E, Tamura H, et al. (1994) Assessment of therapeutic potential of interleukin 2 for myelodysplastic syndromes. $\mathrm{Br} \mathrm{J}$ Haematol 86: 562-567.

32. Kordasti SY, Ingram W, Hayden J, Darling D, Barber L, et al. (2007) CD4+CD25highFoxp3+ regulatory $\mathrm{T}$ cells in myelodysplastic syndrome (MDS). Blood 110: 847-850.

33. Kondo A, Yamashita T, Tamura H, Zhao W, Tsuji T, et al. (2010) Interferon-gamma and tumor necrosis factor-alpha induce an immunoinhibitory molecule, B7-H1, via nuclear factor-kappaB activation in blasts in myelodysplastic syndromes. Blood 116: 1124-1131.

34. Mailloux AW, Sugimori C, Komrokji RS, Yang L, Maciejewski JP, et al. (2012) Expansion of effector memory regulatory T-cells represents a novel prognostic factor in lower risk myelodysplastic syndrome. J Immunol 189: 3198-3208.

35. Lichtman MA (2001) The stem cell in the pathogenesis and treatment of myelogenous leukemia: a perspective. Leukemia 15: 1489-1494.

36. Corey SJ, Minden MD, Barber DL, Kantarjian H, Wang JC, et al. (2007) Myelodysplastic syndromes: the complexity of stem-cell diseases. Nat Rev Cancer 7: 118-129.

37. Mehrotra BB, George TI, Kavanau K, Avet-Loiseau H, Moore II D, et al. (1995) Cytogenetically aberrant cells in the stem cell compartment (CD34+lin-) in acute myeloid leukemia. Blood 86: 1139-1147.

38. Fagioli F, Cuneo A, Carli MG, Bardi A, Piva N, et al. (1993) Chromosome aberrations in CD34-positive acute myeloid leukemia. Correlation with clinicopathologic features. Cancer Genet Cytogenet 71: 119-124.

39. Haase D, Feuring-Buske M, Könemann S, Fonatsch C, Troff C, et al. (1995) Evidence for malignant transformation in acute myeloid leukemia 
Citation: Bandara WMMS, Rathnayake AJIS, Goonasekera HWW, Dissanayake VHW (2016) Development of Cytogenetic Abnormalities in Myelodysplastic Syndromes. J Mol Genet Med 10: 217. doi:10.4172/1747-0862.1000217

Page 5 of 5

at the level of early hematopoietic stem cells by cytogenetic analysis of CD34+ subpopulations. Blood 15: 2906-2912.

40. Nilsson L, Astrand-Grundström I, Anderson K, Arvidsson I, Hokland P, et al. (2002) Involvement and functional impairment of the CD34(+)CD38(-) Thy-1(+) hematopoietic stem cell pool in myelodysplastic syndromes with trisomy 8. Blood 100: 259-267.

41. Jaju RJ, Jones M, Boultwood J, Kelly S, Mason DY, et al. (2000) Combined immunophenotyping and FISH identifies the involvement of B-cells in 5q-syndrome. Gene Chromosome Cancer 29: 276-280.

42. Thanopoulou E, Cashman J, Kakagianne T, Eaves A, Zoumbos N, et al (2004) Engraftment of NOD/SCID-beta2 microglobulin null mice with multilineage neoplastic cells from patients with myelodysplastic syndrome. Blood 103: 4285-4293.

43. Nilsson L, Astrand-Grundstrom I, Arvidsson I, Jacobson B, HellstromLindberg E, et al. (2000) Isolation and characterization of hematopoietic progenitor/stem cells in 5q-deleted myelodysplastic syndromes: evidence for involvement at the hematopoietic stem cell level. Blood 96: 2012-2021.

44. Blau O, Baldus CD, Hofmann WK, Thiel G, Nolte F, et al. (2011) Mesenchymal stromal cells of myelodysplastic syndrome and acute myeloid leukemia patients have distinct genetic abnormalities compared with leukemic blasts. Blood 2011: 118-120.
45. Kim Y, Jekarl DW, Kim J, Kwon A, Choi H, et al. (2015) Genetic and epigenetic alterations of bone marrow stromal cells in myelodysplastic syndrome and acute myeloid leukemia patients. Stem Cell Res 14: 177-184.

46. Song LX, Guo J, He Q, Yang LP, Gu SC, et al. (2012) Bone marrow mesenchymal stem cells in myelodysplastic syndromes: cytogenetic characterization. Acta Haematol 128: 170-177.

47. Tennant GB, Walsh V, Truran LN, Edwards P, Mills KI, et al. (2000) Abnormalities of adherent layers grown from bone marrow of patients with myelodysplasia. Br J Haematol 111: 853-862.

48. Lopez-Villar O, Garcia JL, Sanchez-Guijo FM, Robledo C, Villaron EM, et al. (2009) Both expanded and uncultured mesenchymal stem cells from MDS patients are genomically abnormal, showing a specific genetic profile for the 5q- syndrome. Leukemia 23: 664-672.

49. Ramakrishnan A, Awaya N, Bryant E, Torok-Storb B (2006) The stromal component of the marrow microenvironment is not derived from the malignant clone in MDS. Blood 772-773.

50. Kramer A, Neben K, Ho AD (2001) Centrosome replication, genomic instability and cancer. Leukemia 16: 767-775. 